\title{
MULTIPLE INTEGRATION IS INTRACTABLE BUT NOT HOPELESS
}

\author{
IAN H. SLOAN ${ }^{1}$
}

(Received 16 March 1998)

\section{Introduction}

Multiple integrals in ten or twenty variables are often needed by atomic, molecular and nuclear physicists, because of the large number of degrees of freedom in the quantum systems with which they must deal. In statistics too there is often a need to evaluate integrals with many degrees of freedom. It is in mathematical finance, however, that the most striking examples are seen, with claims of integrals being evaluated during recent years with many hundreds of variables.

The aim of this paper is to explore in a special context some of the mathematical and computational issues that can arise when the dimensionality of multiple integrals is large. As a starting point, we suppose that the integral has already been transformed to one over the $s$-dimensional unit cube,

$$
\text { If }=\int_{[0,1]^{s}} f\left(x^{1}, \ldots, x^{s}\right) d x^{1} \ldots d x^{s}=\int_{[0,1]^{s}} f(x) d x .
$$

Moreover, we suppose that suitable transformations have been carried out so that the integrand $f$ has a continuous 1-periodic extension with respect to each component $x^{k}$, and has Fourier coefficients satisfying, for some $\alpha>1$,

$$
|\hat{f}(h)| \leq \frac{1}{\left(\bar{h}_{1} \ldots \bar{h}_{s}\right)^{\alpha}}, \quad h \in \mathbb{Z}^{s},
$$

where

$$
\hat{f}(h)=\int_{[0,1]^{3}} f(x) e^{-2 \pi i h \cdot x} d x
$$

\footnotetext{
'School of Mathematics, University of New South Wales, Sydney 2052, Australia.

(C) Australian Mathematical Society 2000, Serial-fee code 0334-2700/00
} 
and

$$
\bar{h}= \begin{cases}|h| & \text { if }|h| \geq 1, \\ 1 & \text { if } h=0\end{cases}
$$

This is the most commonly used setting for the study of number-theoretic and lattice rules; for a discussion, see [4].

The integral in (1) is to be approximated by an $N$-point quadrature sum

$$
Q f=\sum_{j=1}^{N} w_{j} f\left(x_{j}\right),
$$

with

$$
x_{j} \in[0,1)^{s}, \quad w_{j} \in \mathbb{R}, \quad j=1, \ldots, N .
$$

\section{A pessimistic result}

In recent joint work with $\mathrm{H}$. Wozniakowski, see [5], a remarkably pessimistic result has been established for the 'worst-case' error as $s \rightarrow \infty$, in the setting indicated above. Roughly speaking, this result says that in order to guarantee an error smaller than the integral itself, one needs to take at least $2^{s}$ quadrature points! Because the rate of growth in the cost is greater than any polynomial in $s$, the quadrature problem in this setting is said to be 'intractable'.

To state the result more precisely, we need some notation. First, for given $\alpha>1$ let $E_{\alpha}$ denote the set of functions $f$ whose Fourier coefficients satisfy the bound (2). For a particular quadrature rule $Q$, let $P_{\alpha}(Q)$ denote the worst-case error for $f \in E_{\alpha}$,

$$
P_{\alpha}(Q):=\sup \left\{|Q f-I f|: f \in E_{\alpha}\right\}
$$

By minimising $P_{\alpha}(Q)$ over all $N$-point quadrature rules we obtain

$$
e(N, s):=\inf \left\{P_{\alpha}(Q): w_{1}, \ldots, w_{N} \in \mathbb{R}, x_{1}, \ldots, x_{N} \in[0,1)^{s}\right\},
$$

which we may think of as the worst-case error for the best $N$-point quadrature rule.

THEOREM 1 ([5]). If $N<2^{s}$ then for any choice of $N$-point quadrature rule $Q$,

$$
e(N, s)=e(0, s)=1 \text {. }
$$


Here $e(0, s)$ denotes the worst-case error with no quadrature points, that is, $e(0, s)=\sup |I f|=\sup |\hat{f}(0)|=1$ for $f \in E_{\alpha}$. The theorem may be interpreted as saying, in effect, that for $N<2^{s}$ the safest course is to take all of the weights in the $N$-point quadrature rule to be zero!

The proof of the theorem proceeds by construction, as follows. Suppose $N<2^{s}$ and assume that an $N$-point quadrature rule $Q$ is given. Then we show that a function $f^{*} \in E_{\alpha}$ can be found such that $I f^{*}=1$ and yet $Q f^{*}=0$. This is enough to prove the results, since from this follows $P_{\alpha}(Q) \geq\left|Q f^{*}-I f^{*}\right|=1$; and since this holds for arbitrary $Q$, it follows that $e(N, s) \geq 1$. Of course $e(N, s) \leq e(0, s)=1$ is trivial, so we will have proved $e(N, s)=e(0, s)=1$.

We seek our factor $f^{*}$ in the form of a trigonometric polynomial,

$$
f^{*}(x)=\theta(x) \sum_{h \in B_{s}} a_{h} e^{2 \pi i h \cdot x},
$$

where

$$
B_{s}=\left\{\left(h_{1}, \ldots, h_{s}\right): h_{k}=0 \text { or } 1, k=1, \ldots, s\right\}
$$

with $\theta$ a polynomial yet to be chosen, and the coefficients $a_{h}$, not all zero, chosen so that

$$
\sum_{h \in B_{s}} a_{h} e^{2 \pi i h \cdot x_{j}}=0, j=1, \ldots, N
$$

The mathematical heart of the argument is just the elementary fact that this homogeneous linear system has a smaller number of equations (namely $N$ ) than unknowns (namely $2^{s}$ ), and therefore certainly has a non-trivial solution. Note that by satisfying (3) we ensure $f^{*}\left(x_{j}\right)=0$ for $j=1, \ldots, N$, so already we ensure $Q f^{*}=0$.

To complete the argument we choose $h^{*} \in B_{s}$ to maximise $\left|a_{h}\right|$, and at the same time normalise the solution of the homogeneous linear system, so that for all $h \in B_{s}$

$$
\left|a_{h}\right| \leq\left|a_{h^{*}}\right|, \quad a_{h^{*}}=1
$$

Then $\theta$ is defined by $\theta(x)=e^{-2 \pi i h^{*} \cdot x}$, implying

$$
f^{*}(x)=\sum_{h \in B_{s}} a_{h} e^{2 \pi i\left(h-h^{*}\right) \cdot x}
$$

and hence $I f^{*}=a_{h^{*}}=1$. It is clear that $f^{*} \in E_{\alpha}$, because $\left|a_{h}\right| \leq 1$ and the components of $h-h^{*}$ are all 1,0 or -1 , thus the proof is complete. 


\section{Lattice rules with multiples of $2^{s}$ points}

We have just seen that, in this particular setting at least, rules with fewer than $2^{s}$ points are destined to fail, in the worst-case sense. It is therefore interesting that once one reaches that magic number of $2^{s}$ quadrature points the situation changes completely, in that there is a particular rule, namely the product-rectangle rule $R_{2}$ (which is the equal-weight rule obtained by taking a tensor product of 2-point (left) rectangle rules), whose worst-case error for suitable integrals can be much less than 1 . Indeed, it can easily be shown, by the methods indicated below, that

$$
P_{\alpha}\left(R_{2}\right)=\left(1+\frac{\zeta(\alpha)}{2^{\alpha-1}}\right)^{s}-1,
$$

where $\zeta(\alpha)$ is the Riemann zeta function, thus $P_{\alpha}\left(R_{2}\right)$ converges to zero as $\alpha \rightarrow \infty$. Yet for every value of $\alpha$ and any rule $Q$ we have, for $N<2^{s}$,

$$
P_{\alpha}(Q) \geq e(N, s) \geq 1 .
$$

The rectangle rule $R_{2}$ is one of the simplest examples of what are known as 'lattice rules'. It is useful to introduce lattice rules in this context, because there are some lattice rules that do possess reasonable properties for $N \geq 2^{s}$.

By definition, a lattice rule is an equal-weight quadrature rule

$$
Q f=\frac{1}{N} \sum_{j=0}^{N-1} f\left(x_{j}\right)
$$

where $x_{0}, \ldots, x_{N-1}$ are all the points of an 'integration lattice' that lie in $[0,1)^{s}$. An integration lattice in $\mathbb{R}^{s}$ is a discrete subset of $\mathbb{R}^{s}$ that is closed under addition and subtraction (and so is a group under addition) and that contains $\mathbb{Z}^{s}$ as a subset.

For a full discussion of lattice rules, see [4]. For present purposes there are just a few points we need to emphasize. The first is that for the lattice rule $Q$ corresponding to the integration lattice $L$ there is a simple expression for the worst-case error in $E_{\alpha}$, namely

$$
P_{\alpha}(Q)=\sum_{h \in L^{\perp}}^{\prime} \frac{1}{\left(\bar{h}_{1} \ldots \bar{h}_{s}\right)^{\alpha}}
$$

where $L^{\perp}$ is the so-called 'dual lattice' of $L$, defined by

$$
L^{\perp}=\left\{h \in \mathbb{Z}^{s}: x \cdot h \in \mathbb{Z} \forall x \in L\right\},
$$

and where the prime indicates that the point $h=0$ is to be omitted from the sum. 
Next, we want to define the classical kind of lattice rule, namely the 'numbertheoretic' rules (nowadays called 'lattice rules of rank 1'), which are rules of the form

$$
S_{m} f=\frac{1}{m} \sum_{j=0}^{m-1} f\left(j \frac{z}{m}\right),
$$

where $z$ is a (carefully chosen) point in $\mathbb{Z}^{s}$.

There exists an extensive theory of rank-1 lattice rules. One of the earliest results, due to Bahvalov in 1959 [1], says that if $N$ is prime then there exists an $N$-point rank-1 lattice rule $S_{N}$ such that

$$
P_{\alpha}\left(S_{N}\right) \leq C(s, \alpha) \frac{(\log N)^{\alpha(s-1)}}{N^{\alpha}},
$$

with the 'constant' $C(s, \alpha)$ not specified. A recent result, due to [3], which gives more detailed information while preserving the order of convergence with respect to $N$, is

$$
P_{\alpha}\left(S_{N}\right) \leq 2 \zeta(s-1) \alpha \frac{2^{\alpha(s-1)}}{((s-1) !)^{\alpha}}\left(\frac{\alpha}{\alpha-1}\right)^{\alpha} \frac{(\log N)^{\alpha(s-1)}}{N^{\alpha}}(1+o(1))
$$

as $N \rightarrow \infty$.

Finally, we want to define rules which are ' $2^{s}$ copies of rank-1 rules'. Explicitly, the unique rule which is a $2^{s}$ copy of the $m$-point rank-1 rule defined in (4) is

$$
C_{2^{s} m} f=\frac{1}{2^{s} m} \sum_{k_{1}=0}^{1} \ldots \sum_{k_{s}=0}^{1} \sum_{j=0}^{m} f\left(\frac{k}{2}+\frac{j z}{2 m}\right) .
$$

This too is a lattice rule. Moreover it is a rule which has better behaviour than a classical rank-1 rule, at least on the average, according to the following theorem of [2].

THEOREM 2. Let $m$ be prime, and let $N$ be a prime number closest to $2^{s} m$. Then

$$
\frac{\text { mean } P_{\alpha}\left(C_{2^{s}}\right)}{\text { mean } P_{\alpha}\left(S_{N}\right)} \leq\left(\frac{2+\zeta(\alpha) / 2^{\alpha-2}}{1+2 \zeta(\alpha)}\right)^{s}
$$

Here mean $P_{\alpha}\left(S_{N}\right)$ is the mean of $P_{\alpha}\left(S_{N}\right)$ taken over all $z$-vectors in (4) (with $m$ replaced by $N$ ) whose components satisfy

$$
-\frac{N}{2}<z_{k} \leq \frac{N}{2}, \quad z_{k} \neq 0, \text { for } k=1, \ldots, s,
$$


while mean $P_{\alpha}\left(C_{2^{s} m}\right)$ is the mean of $P_{\alpha}\left(C_{2^{s} m}\right)$ taken over all $z$-vectors in (5) with components satisfying

$$
-\frac{m}{2}<z_{k} \leq \frac{m}{2}, \quad z_{k} \neq 0, \text { for } k=1, \ldots, s .
$$

Lattice rules which are $2^{s}$ copies of rank- 1 lattice rules also have practical virtues: firstly, good examples are relatively easy to find; secondly, they can be implemented in an efficient manner; and thirdly, an a posteriori error estimate can be obtained at the same time as the estimate of the integral at essentially no cost. For all these aspects of copy rules the reader is referred to [4].

\section{Acknowledgement}

The continuing support of the Australian Research Council is gratefully acknowledged.

\section{References}

[1] N. S. Bahvalov, "On approximate calculation of multiple integrals", Vestnik Moskovskogo Universiteta, Seriya Matematiki, Mehaniki, Astronomii, Fiziki, Himii 4 (1959) 3-18, (Russian).

[2] S. A. R. Disney and I. H. Sloan, "Lattice integration rules of maximal rank formed by copying rank 1 rules", SIAM Journal on Numerical Analysis 29 (1992) 566-77.

[3] H. Niederreiter, "Improved error bounds for lattice rules", Joumal of Complexity 9 (1993) 60-75.

[4] I. H. Sloan and S. Joe, Lattice Methods for Multiple Integration (Oxford University Press, 1994).

[5] I. H. Sloan and H. Wozniakowski, "An intractability result for multiple integration", Mathematics of Computation 66 (1997) 1119-1124. 